\title{
Energetics of Pore Formation Induced by Membrane Active Peptides ${ }^{\dagger}$
}

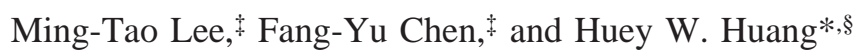 \\ Department of Physics, National Central University, Chung-Li 32054, Taiwan, and Department of Physics \& Astronomy, \\ Rice University, Houston, Texas 77251
}

Received December 1, 2003; Revised Manuscript Received January 15, 2004

\begin{abstract}
Antimicrobial peptides are known to form pores in cell membranes. We study this process in model bilayers of various lipid compositions. We use two of the best-studied peptides, alamethicin and melittin, to represent peptides making two types of pores, that is, barrel-stave pores and toroidal pores. In both cases, the key control variable is the concentration of the bound peptides in the lipid bilayers (expressed in the peptide-lipid molar ratio, $P / L$ ). The method of oriented circular dichroism $(\mathrm{OCD})$ was used to monitor the peptide orientation in bilayers as a function of $P / L$. The same samples were scanned by $\mathrm{X}$-ray diffraction to measure the bilayer thickness. In all cases, the bilayer thickness decreases linearly with $P / L$ and then levels off after $P / L$ exceeds a lipid-dependent critical value, $(P / L)^{*}$. OCD spectra showed that the helical peptides are oriented parallel to the bilayers as long as $P / L<(P / L)^{*}$, but as $P / L$ increases over $(P / L)^{*}$, an increasing fraction of peptides changed orientation to become perpendicular to the bilayer. We analyzed the data by assuming an internal membrane tension associated with the membrane thinning. The free energy containing this tension term leads to a relation explaining the $P / L$-dependence observed in the OCD and X-ray diffraction measurements. We extracted the experimental parameters from this thermodynamic relation. We believe that they are the quantities that characterize the peptide-lipid interactions related to the mechanism of pore formation. We discuss the meaning of these parameters and compare their values for different lipids and for the two different types of pores. These experimental parameters are useful for further molecular analysis and are excellent targets for molecular dynamic simulation studies.
\end{abstract}

Membrane active peptides, including antimicrobials and toxins, are known to induce transmembrane pores. The first peptide discovered to do so is alamethicin $(1,2)$. At first, alamethicin was thought to induce pores (which were detected by ion conduction) only by a transmembrane electric potential (see review in ref 3 ). However, numerous experiments $(4-8)$ indicated that alamethicin could insert into bilayers in the absence of an external field (see review in ref 9). Although it was believed that alamethicin insertion would create pores, a direct correlation with ion conduction was difficult to establish. Later, with the combination of oriented circular dichroism $(10,11)$ and neutron diffraction $(12,13)$, we showed the direct correlation between alamethicin insertion (without voltage) and transmembrane pores. Two other extensively studied peptides, bee venom toxin melittin (14) and frog peptide magainin (15), also exhibited similar behaviors. Pores were evidently formed by both melittin $(16-18)$ and magainin $(19,20)$ because they caused leakage of fluorescent dyes from lipid vesicles. In the last 15 years, a great variety of antimicrobial peptides have been shown to induce transmembrane pores in bacterial

† This work was supported by NIH Grants GM55203 and RR14812 and by the Robert A. Welch Foundation (to H.W.H) and by National Science Council (Taiwan) through Contract NSC92-2112-M-008-013 (to F.-Y.C.).

* To whom correspondence should be addressed. Tel: 7133484899 . Fax: 713 3484150. E-mail: hwhuang@ rice.edu.

$\stackrel{*}{*}$ National Central University.

$\S$ Rice University. cells as well as in lipid vesicles (see reviews in refs 21 and 22-24). Understanding the mechanism of pore formation induced by peptides will provide insights into the functions of antimicrobial peptides, which are essential components of the innate immune system, and facilitate the development of new anti-infective therapeutics. Pore formation is also potentially useful for gene and drug deliveries (24).

To understand how peptides induce pore formation, consider the simpler case of pores in pure lipid bilayers, which have been extensively studied both experimentally and theoretically $(25-31)$. In pure lipid bilayers, pores are always produced under tension. The initiation of a pore is a dynamic process that is difficult to analyze, because it often involves nucleation defects $(28,30,32)$. However, once a pore is formed, its essential mechanics is well understood. A pore in a pure lipid bilayer is governed by the energy $E_{R}$, which is defined as the energy difference between a bilayer with a circular pore of radius $R$ and a bilayer without a pore (25, 26):

$$
E_{\mathrm{R}}=\gamma 2 \pi R-\sigma \pi R^{2}
$$

The first term represents the free energy cost of creating the rim or the edge of the pore; $\gamma$ is the line tension, or the energy cost per unit length of the edge. The second term represents the (negative) work done by the membrane tension $\sigma$ to create a pore of area $\pi R^{2}$. The driving force for pore opening is the membrane tension, while that for closure is the line tension. For given $\gamma$ and $\sigma, E_{\mathrm{R}}$ is maximum at $R=\gamma / \sigma$. 
Thus a pore in a pure lipid bilayer is unstable: a pore of radius smaller than $\gamma / \sigma$ tends to close, whereas a pore of radius larger than $\gamma / \sigma$ tends to expand indefinitely. This general behavior of pores in pure lipid bilayers suggests that the pore-inducing peptides must (1) create a stress in the membrane equivalent to a membrane tension to open pores and (2) also stabilize the pores once produced.

In previous publications $(33,34)$, we have presented experimental evidence and thermodynamic arguments for the tension effect. Here we will provide a qualitative argument for the stability of the peptide-induced pores. The main purpose of this paper is to present experimental parameters of lipid-peptide interactions that underline the cause and the effect of the peptide-induced tension. We will discuss the meaning of these parameters and show that the interactions depend on the size of lipid headgroup and the chain cross section. We will also show how the interactions are different between toroidal pores and barrel-stave pores (35). These experimental parameters provide excellent targets for molecular dynamics simulations.

Among the known antimicrobials, only alamethicin and its analogues have been shown to form barrel-stave pores. Melittin, magainin, protegrin, and perhaps most cationic antimicrobial peptides form toroidal pores (35). In this paper, alamethicin and melittin are used to study these two types of pores. Alamethicin and melittin are the most studied peptides and hence have the most complete experimental data, including that of single crystals.

\section{MATERIALS AND METHODS}

Materials. 1,2-Diphytanoyl-sn-glycero-3-phosphocholine (DPhPC), ${ }^{1}$ 1-palmitoyl-2-oleoyl-sn-glycero-3-phosphocholine (POPC), 1,2-dioleoyl-sn-glycero-3-phosphocholine (DOPC), 1,2-dioleoyl-sn-glycero-3-phosphoethanolamine (DOPE), and 1,2-dierucoyl-sn-glycero-3-phosphocholine (DiC22:1PC) were purchased from Avanti Polar Lipids (Alabaster, AL). Alamethicin and melittin were purchased from Sigma-Aldrich Chemical Co. (St. Louis, MO). Sigma alamethicin is a mixture of components, principally alamethicin I ( $85 \%$ by high-performance liquid chromatography) and alamethicin II (12\%), which differ by one amino acid (36). The same material has been used in all previous studies $(9,11,33,34$, 37,38 ). Two grades of melittin were used, the sequencing grade (product no. M-1407) and the grade of purity 93\% HPLC (product no. M-2272). Both gave the same results in this study. Yang et al. (35) also found no difference between Sigma melittin and pure synthetic melittin in this type of study as long as there was no added $\mathrm{Ca}^{2+}$ in the sample. Poly(ethylene glycol) (PEG20000) was purchased from Merk Co. (Hohenfrunn, Germany). All materials were used as delivered.

Sample Preparation. Two experimental methods were used in this study. One was oriented circular dichroism (OCD; $10,11)$ for the measurement of peptide orientation in lipid

\footnotetext{
${ }^{1}$ Abbreviations: DPhPC, 1,2-diphytanoyl-sn-glycero-3-phosphocholine; POPC, 1-palmitoyl-2-oleoyl-sn-glycero-3-phosphocholine; DOPC, 1,2-dioleoyl-sn-glycero-3-phosphocholine; DOPE, 1,2-dioleoyl-sn-glycero-3-phosphoethanolamine; DiC22:1PC, 1,2-dierucoyl-sn-glycero-3phosphocholine; Ala, alamethicin; Mel, melittin; $P / L$, the molar ratio of the bound peptide to lipid; $(P / L)^{*}$, the threshold (or critical) peptide concentration for pore formation; $\mathrm{OCD}$, oriented circular dichroism; LXD, lamellar X-ray diffraction.
}

bilayers. Another was lamellar X-ray diffraction (LXD) for the measurement of membrane thickness. The samples used in both methods were in the form of oriented multilayers, a stack of parallel lipid bilayers on a solid substrate. The preparation of such oriented samples followed the method described in the previous study (33). Briefly, lipid and peptide of chosen peptide-to-lipid molar ratio $(P / L)$ were codissolved in a solvent of 1:1 (v/v) methanol and chloroform. The lipid concentration was about $1 \mathrm{mg}$ per $20 \mu \mathrm{L}$ of solvent. The solution of appropriate amount was spread onto a cleaned quartz surface, $10 \mu \mathrm{L}$ or less of solution (depending on the $P / L$ ) onto a $14-\mathrm{mm}$ diameter area for OCD or 100 $\mu \mathrm{L}$ of solution onto a $20-\mathrm{mm}$ square area for LXD. When the solvent dried, the sample was vacuumed to remove the remaining solvent residues and then slowly hydrated with water vapor until it appeared transparent. A good sample was visually smooth and showed at least 5 orders of Bragg diffraction by LXD.

OCD Measurement. The procedure of OCD measurement has been described in Chen et al. (33). All sample temperatures were set at $30{ }^{\circ} \mathrm{C}$. A water solution of poly(ethylene glycol) (PEG20000) was inside a sealed sample chamber to control the relative humidity, which in turn set the degree of hydration of the sample. The concentration of PEG solution used in this study was $4.75 \mathrm{~g}$ of PEG20000 in 10.00 $\mathrm{g}$ of water, which gave a vapor pressure equivalent to $98 \%$ relative humidity $(\mathrm{RH})$ at $30^{\circ} \mathrm{C}$. The hydration equilibrium of the sample was ensured by an agreement of at least three OCD spectra measured over a period of $6 \mathrm{~h}$. OCD was measured with a Jasco J-810 spectropolarimeter with light incident normal to the sample surface (11). The background OCD spectra of pure lipid bilayers (i.e., without peptides) were measured separately and were removed from the spectra of the corresponding samples containing peptides.

The reason we chose 98\% RH (rather than 100\% RH) for this experiment was that for both OCD and LXD measurements, the sample substrate was oriented vertically. At levels of humidity higher than $98 \% \mathrm{RH}$, the membranes deposited on one substrate would flow. This is not to say that it is impossible to make measurements at $100 \% \mathrm{RH}$. An oriented membrane sample could be covered with another substrate to prevent the sample flow, as we have done previously for $\operatorname{OCD}(11,33)$ and for $\operatorname{LXD}(37,39)$. However, it would take a long equilibrating time to change the hydration level of a covered (i.e., two-substrate) sample, and hydration changes are necessary in an X-ray experiment for the purpose of phase determination. Our previous experiments mentioned above have shown that the dependence of the peptide transition on hydration is gradual. There is no qualitative difference between the orientation transitions measured at $98 \% \mathrm{RH}$ and at $100 \% \mathrm{RH}(33)$.

The OCD studies of four peptide/lipid systems, Ala/ (DOPC/PE 2:1), Mel/DPhPC, Mel/DiC22:1PC, and Mel/ POPC, are reported here. The results of two other systems, $\mathrm{Ala} / \mathrm{DPhPC}$ and Mel/DOPC, were reported previously (33, 34)

LXD Measurement. The sample chamber for LXD was the same as that used in our previous studies $(40,41)$, except that the relative humidity was controlled by a series of PEG solutions enclosed inside the chamber. This was to ensure that the hydration levels of the samples were the same in the OCD and LXD measurements. The temperature was set 

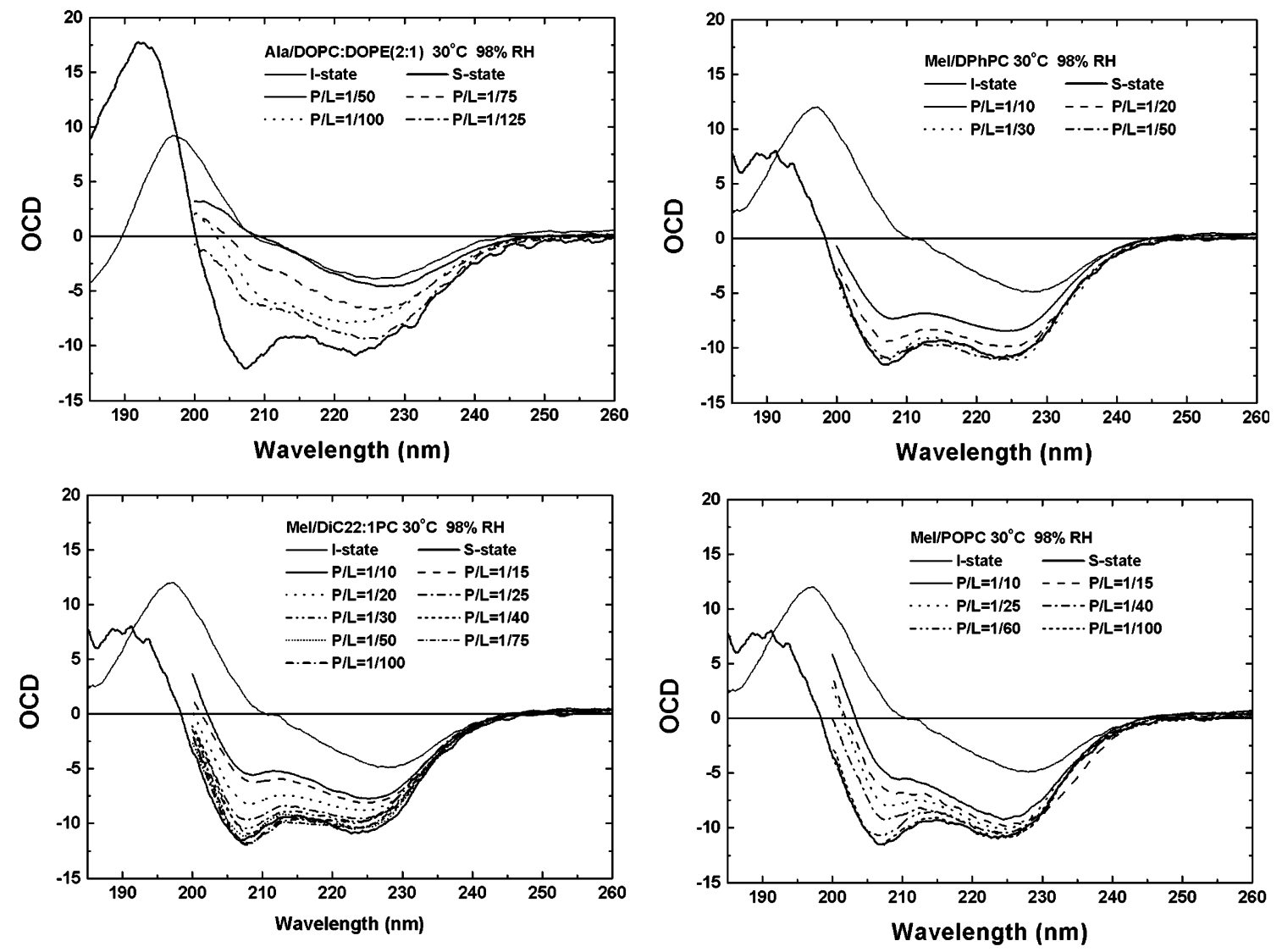

FIGURE 1: OCD spectra of alamethicin in the DOPC/DOPE 2:1 mixture bilayers, melittin in DPhPC bilayers, melittin in DiC22:1PC bilayers, and melittin in POPC bilayers at $30^{\circ} \mathrm{C}$ and $98 \% \mathrm{RH}$. Appropriate lipid background, that is, the OCD of the same amount of lipid, was removed from each spectrum. The $\mathrm{CD}$ amplitudes are in an arbitrary unit. The I and S spectra of alamethicin and melittin were reproduced from data of refs 33 and 34 , respectively. They are the spectra of the helical peptide oriented perpendicular and parallel to the bilayer, respectively. I and S were relatively normalized to each other. High UV absorption by unsaturated lipids made the spectra below $\sim 200 \mathrm{~nm}$ unacceptably noisy. Nevertheless the somewhat incomplete spectra are adequate for spectra fitting to determine the fraction of the peptide molecules in the I (or S) state.

at $30{ }^{\circ} \mathrm{C}$, the same temperature for OCD measurements. In addition to the measurement at $98 \% \mathrm{RH}$, a series of measurement were made at lower levels of humidity for the purpose of phase determination. Precise RH reading for these lower levels of humidity was not necessary because the swelling method for phase determination uses the lamellar repeat spacing as the variable.

LXD was measured with $\mathrm{Cu} \mathrm{K} \alpha$ radiation generated at $30 \mathrm{kV} / 30 \mathrm{~mA}$ by $\theta-2 \theta$ scan from $\theta=0.5-7.5^{\circ}$ with a step size $\Delta \theta=0.01^{\circ}$ at $1 \mathrm{~s}$ per step. The equilibrium of the sample at each humidity setting was ensured by an agreement of at least three consecutive diffraction patterns the average of which was subsequently analyzed. Only samples that produced at least five discernible diffraction peaks were accepted. Each peptide-lipid combination was measured with at least two separately prepared samples. Each sample was measured twice at least $10 \mathrm{~h}$ apart to check the reproducibility.

The procedure for data reduction was described in many of our papers $(37,39-42)$. Briefly, the procedure started with background removal and absorption and diffraction volume corrections. Then the integrated peak intensities were corrected for the polarization and the Lorentz factors. The magnitude of the diffraction amplitude was the square root of the integrated intensity. The phases were determined by the swelling method (43). With their phases determined, the diffraction amplitudes were Fourier transformed to obtain the transbilayer electron density profiles. The profiles were not normalized to the absolute scale, but they gave the correct peak-to-peak distances, since the latter are independent of normalization (37).

LXD measurements of two peptide/lipid systems, Mel/ DiC22:1PC and Mel/POPC, are reported here. Four other systems, Ala/DPhPC, Ala/(DOPC/PE 2:1), Mel/DPhPC, and Mel/DOPC, were published previously $(33,34)$.

\section{RESULTS}

Fraction of Peptide Molecules Oriented Normal to the Bilayer as a Function of P/L. The OCD spectra of alamethicin and melittin have been extensively discussed in previous publications $(33,34)$. We used the same method here to analyze the new results. Figure 1 shows the raw data of OCD measurements for Ala/(DOPC/PE 2:1), Mel/DPhPC, $\mathrm{Mel} / \mathrm{DiC} 22: 1 \mathrm{PC}$, and Mel/POPC systems, each for a series of $P / L$. Lipids with unsaturated bonds exhibited high UV absorption at wavelengths below $200 \mathrm{~nm}$, which made the OCD spectra in that region extremely noisy, and therefore, they are not shown in Figure 1.

Alamethicin and melittin both form helices when bound to lipid bilayers. Each has been found to be oriented either parallel or perpendicular to the plane of the bilayer, depending on the sample condition. In previous experiments, we have carefully obtained the mutually normalized OCD spectra 


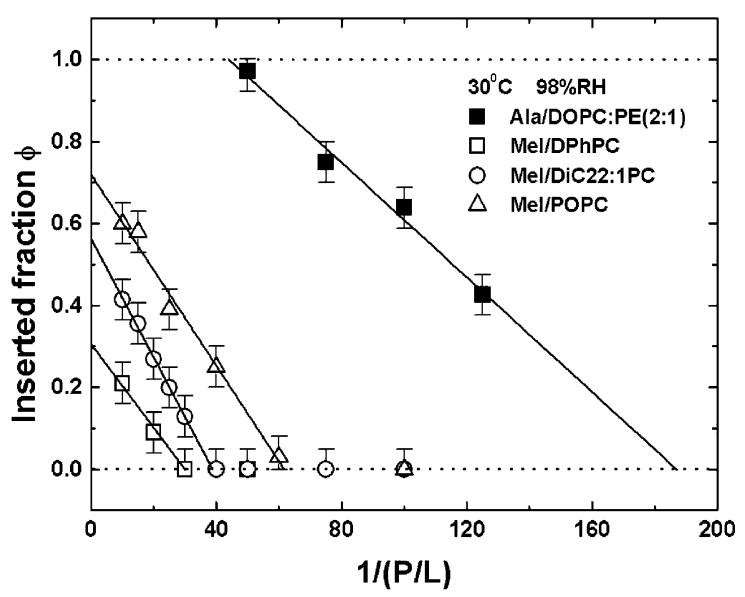

FIGURE 2: Fractions of the peptide molecules in the I state (helices perpendicular to the bilayer), $\phi$, are plotted as a function of the inverse of $P / L$. For each peptide/lipid system, $\phi$ is zero at low values of $P / L$. However, $\phi$ increases linearly with $-1 /(P / L)$ as $P / L$ exceeds a certain threshold value, in agreement with the prediction given by eq 5 . The intercept of the linear fit at the high $P / L$ region with the baseline $\phi=0$ gives the threshold peptide concentration, $(P /$ $L)^{*}$. The slope gives the value $\beta$ according to eq 5 .
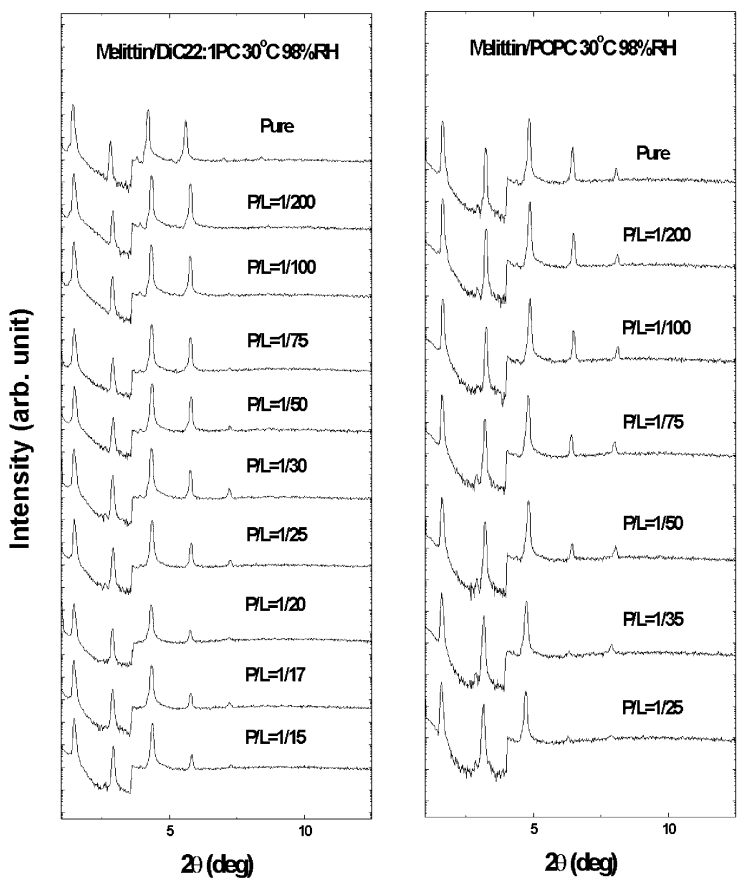

FIGURE 3: X-ray diffraction patterns of melittin/DiC22:1PC and melittin/POPC systems for a series of $P / L$ at the highest hydration level measured. The patterns are displaced for clarity. The steps at $2 \theta \approx 4^{\circ}$ were the results of using an X-ray attenuator that reduced the count rates for the first two diffraction peaks to not saturate the photon counter. Note that each pattern has at least 5 Bragg orders.

for these two orientations by using one sample at different temperatures or RHs, and denoted them as I and S spectra, respectively, for perpendicular and parallel (to the plane of the bilayer) orientations. These standard spectra were reproduced in each panel in Figure 1. Each newly measured OCD spectrum was fitted, after the lipid background removal, with a linear combination of $\mathrm{I}$ and $\mathrm{S}, a \mathrm{I}+b \mathrm{~S}$, and then replotted with the original amplitude multiplied by a factor $1 /(a+b)$ in Figure 1. The fraction of the peptide molecules in the I state (perpendicular to the bilayer) is denoted as $\phi=a /(a+$
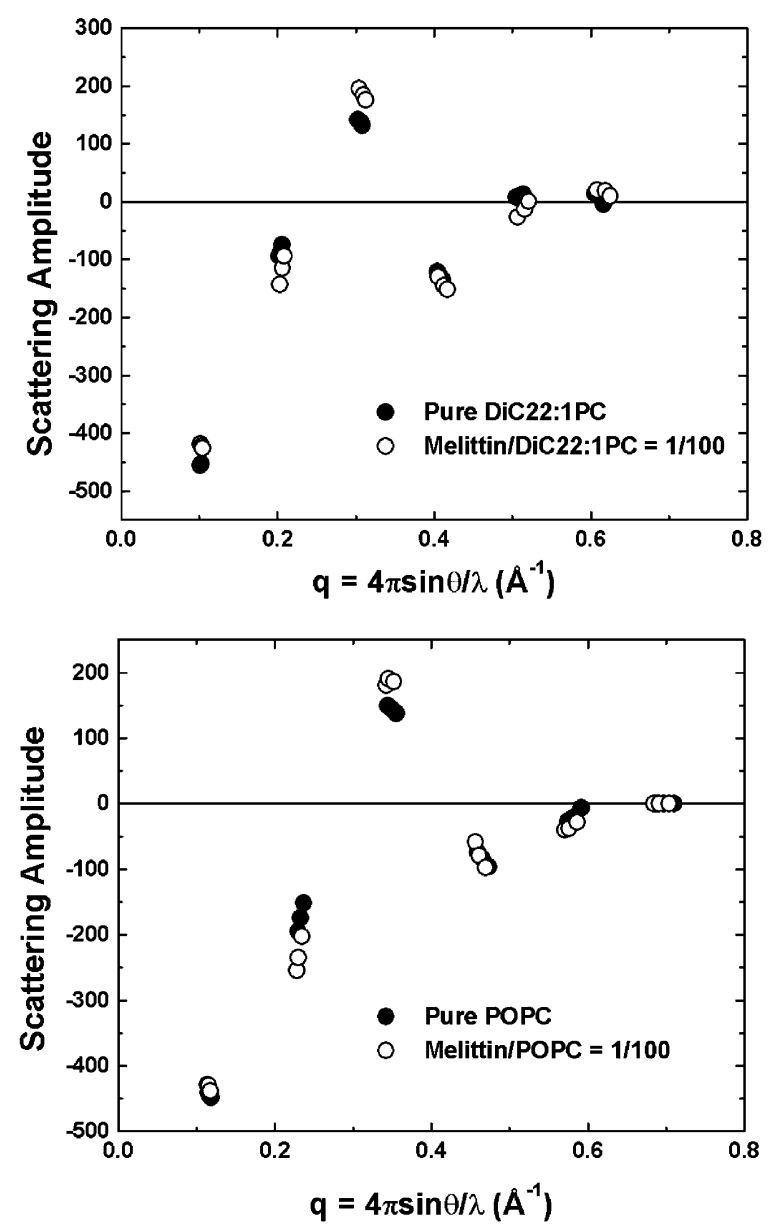

FIGURE 4: Phasing diagrams for the X-ray diffraction of pure DiC22:1PC and DiC22:1PC containing melittin at $P / L=1 / 100$ (top) and of pure POPC and POPC containing melittin at $P / L=1 / 100$ (bottom). The abscissa is the X-ray momentum transfer $q=4 \pi$ $\sin \theta / \lambda$, where $\lambda$ is the $\mathrm{X}$-ray wavelength, $1.54 \AA$. The phases were chosen according to the swelling method $(43,45,46)$.

b). $\phi$ is plotted as a function of $1 /(P / L)$ for the four peptide/ lipid systems in Figure 2.

Membrane Thickness as a Function of P/L. Diffraction patterns of Mel/DiC22:1PC and Mel/POPC systems are shown in Figure 3 for a series of $P / L$ at the highest $\mathrm{RH}$ measured. The complete data include two other sets of diffraction patterns measured at lower $\mathrm{RH}$ for the purpose of phasing. Each pattern has at least 5 Bragg orders. No peak broadening with order was observed, indicating that the undulation fluctuations were negligible at hydration levels below 98\% RH (44). After the data reduction (see Materials and Methods), each sample has three sets of diffraction amplitudes at three different repeat spacings. For the purpose of phase determination, the amplitudes were relatively normalized according to the Blaurock method (43) and plotted against the scattering momentum, $q$. Four examples are shown in Figure 4, where the phases were chosen according to the swelling principle $(45,46)$.

With the phases determined, the diffraction amplitudes were Fourier transformed to the transbilayer electron density profiles shown in Figure 5. We then plotted the peak-topeak (PtP) spacing against $P / L$ for each sample (Figure 6 ). The error bars in Figure 6 represented the ranges of reproducibility of four measurements, two measurements for each of two separately prepared samples. The hydrocarbon 

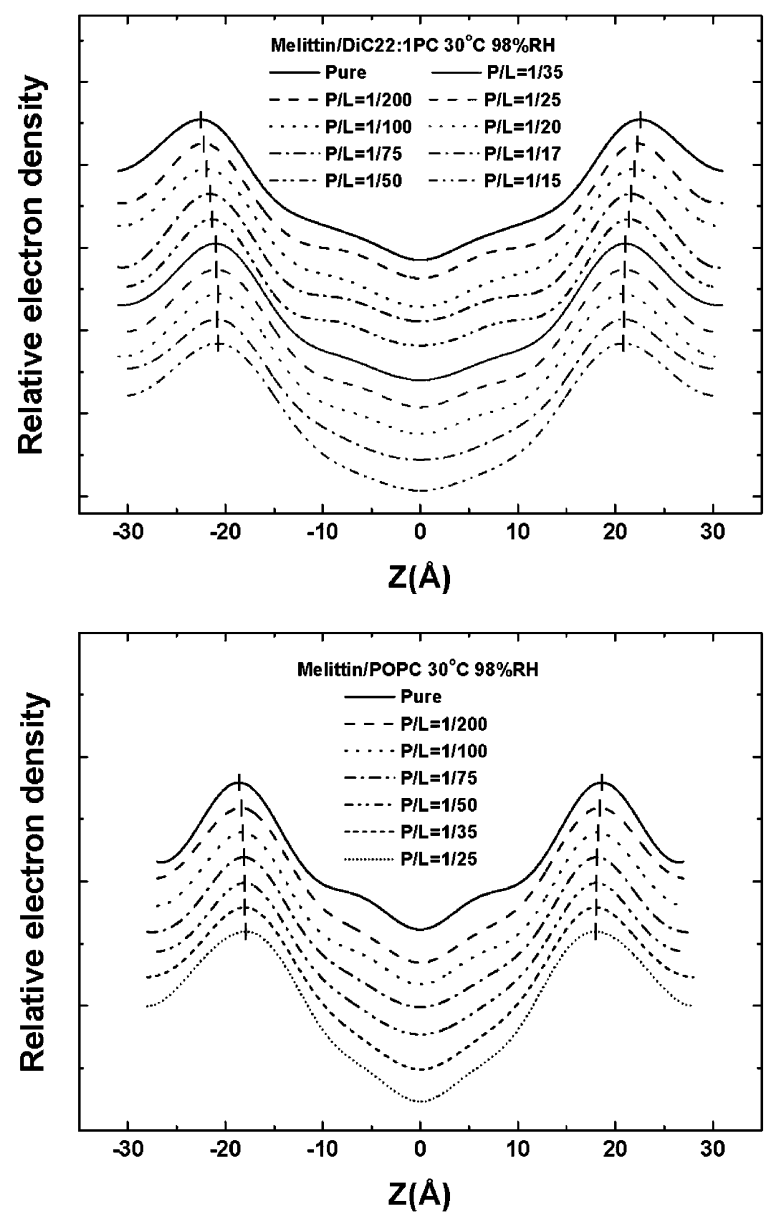

FIGURE 5: Electron density profiles of melittin/DiC22:1PC systems for a series of $P / L$ from 0 to $1 / 15$ (top) and of melittin/POPC systems for a series of $P / L$ from 0 to $1 / 25$ (bottom). The profiles are not normalized and are displaced for clarity. The short vertical bars indicate the positions of the peaks from which the peak-topeak distances, PtP, were measured.

thickness, $h$, of the bilayer was estimated by subtracting twice the length of the glycerol region (from the phosphate to first methylene of the hydrocarbon chain), that is, $\sim 10 \AA$, from PtP (47-51).

\section{EXPERIMENTAL PARAMETERS}

Table 1 was compiled from the OCD and LXD data above. In addition, we also included the parameters calculated from the published OCD data for Ala/DPhPC and Mel/DOPC and the published LXD data for Ala/DPhPC, Ala/(DOPC/PE 2:1), $\mathrm{Mel} / \mathrm{DPhPC}$, and Mel/DOPC $(33,34)$. The bilayer stretch moduli, $K_{\mathrm{A}}$ 's, were measured by Rawicz et al. (52) for more than 10 lipids using the vesicle aspiration method. Surprisingly the $K_{\mathrm{A}}$ 's are all about $240 \mathrm{pN} / \mathrm{nm}$ within experimental errors. Hence we use this average value for our discussion. The hydrocarbon thickness $h$ of each pure lipid bilayer was calculated from its PtP by subtracting twice the length of the glycerol region (10 $\AA$ ) as mentioned in the Results section. The lipid area cross section, $A_{\mathrm{L}}$, was calculated from the hydrocarbon volume (48) of each lipid divided by its $h$ value.

1. Area Expansion per Peptide, $A_{P}$. The idea that peptide binding creates membrane tension was derived from the observation that every antimicrobial peptide that we investigated caused membrane thinning. The peptides that we have
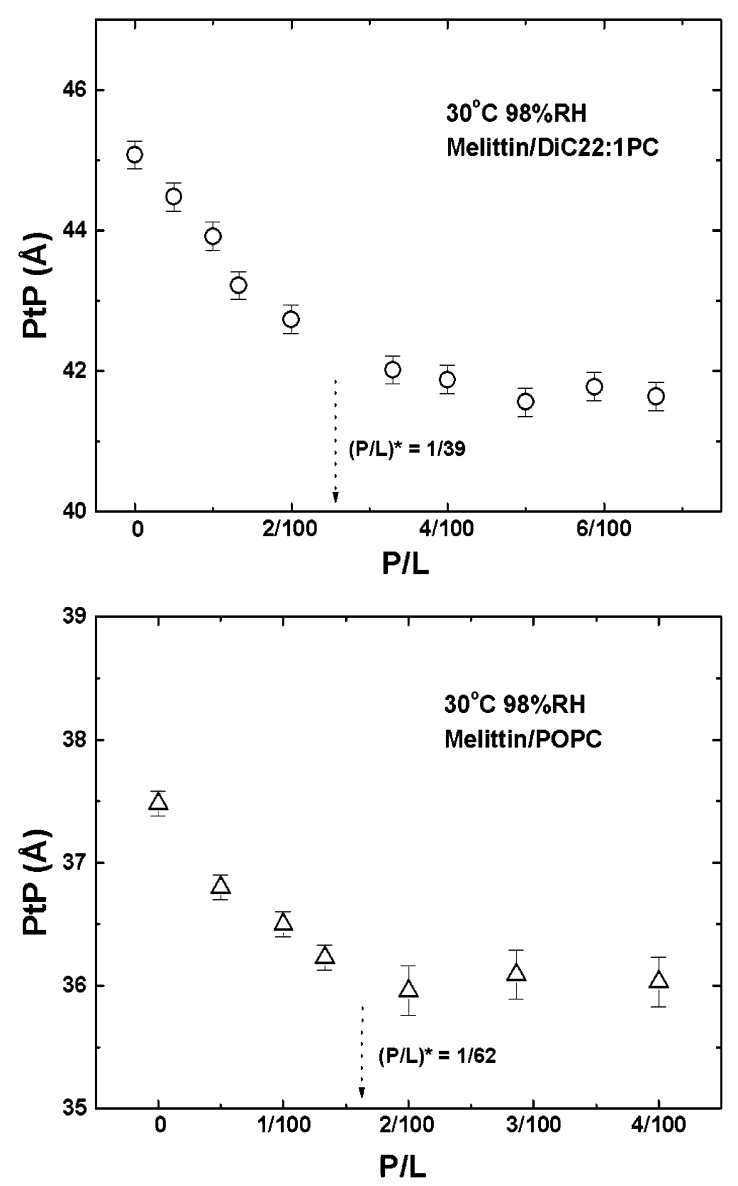

FIGURE 6: Peak-to-peak distance (PtP) versus $P / L$ for melittin in DiC22:1PC (top) and for melittin in POPC (bottom). The error bars represent the ranges of reproducibility by four measurements. The arrows indicate $(P / L) *$ determined by the OCD measurement (see Figure 2). PtP decreases linearly with $P / L$ below $(P / L)^{*}$ and is constant above $(P / L)^{*}$ within the experimental errors.

investigated include alamethicin (37), magainin (42), protegrin (53), melittin (34), and many of their analogues. (One exception is $\theta$-defensin the thinning effect of which is 1 order of magnitude smaller compared with the aforementioned peptides (54). Interestingly, its mechanism is also different (55-57)). We assumed that the membrane thinning is caused by the peptides stretching the membrane area, which is the direct result of the peptide molecules being embedded in the headgroup region (see the cartoon in Figure 7). Other experiments supporting the peptide embedment in the headgroup region include solid-state NMR $(58,59)$, Raman (60), fluorescence (61), differential scanning calorimetry (DSC, $62)$, and titration calorimetry $(63,64)$. A membrane area expansion caused by melittin adsorption has also been observed by vesicle aspiration (65) at constant vesicle volume, while no permeation through the membrane occurred. We have argued, on the basis of the theory of membrane elasticity (66), that the peptide molecules embedded in the headgroup region are dispersed rather than aggregated. This was indeed supported by evidence from fluorescence energy transfer (67-69), NMR (58), and electron paramagnetic resonance (EPR, 70) studies. Thus the fractional increase of the monolayer area due to peptide binding is $\triangle A / A=\Delta A_{\mathrm{L}} / A_{\mathrm{L}}=\left(A_{\mathrm{P}} / A_{\mathrm{L}}\right)(P / L)$, where $P / L$ is the bound peptide-to-lipid molar ratio, $A_{\mathrm{L}}$ the area cross section per lipid, and $A_{\mathrm{P}}$ the area increase caused by one 


\begin{tabular}{|c|c|c|c|c|c|c|}
\hline \multirow{2}{*}{$\begin{array}{c}\text { peptide: } \\
\text { lipid: }\end{array}$} & \multicolumn{4}{|c|}{ melittin } & \multicolumn{2}{|c|}{ alamethicin } \\
\hline & $\overline{\mathrm{DPhPC}}$ & DOPC & DiC22:1PC & $\overline{\text { POPC }}$ & $\overline{\mathrm{DPhPC}}$ & DOPC:PE(2:1) \\
\hline$K_{\mathrm{A}}(\mathrm{pN} / \mathrm{nm})$ & 240 & 240 & 240 & 240 & 240 & 240 \\
\hline$h(\AA)$ & 26.2 & 26.6 & 35.1 & 27.5 & 26.2 & 27.7 \\
\hline$A_{\mathrm{L}}\left(\AA^{2}\right)$ & 91 & 74 & 69 & 68 & 91 & 71 \\
\hline$A_{\mathrm{P}}\left(\AA^{2}\right)$ & 175 & 246 & 237 & 223 & 193 & 208 \\
\hline$(P / L)^{*}$ & $1 / 30$ & $1 / 99$ & $1 / 39$ & $1 / 62$ & $1 / 58$ & $1 / 187$ \\
\hline$\beta$ & -2.27 & -0.95 & -0.77 & -0.39 & 0.24 & 0.24 \\
\hline$\delta \in(\mathrm{kcal} / \mathrm{mol})$ & 12.7 & 5.6 & 12.8 & 5.7 & 1.9 & 0.9 \\
\hline$\sigma^{*}(\mathrm{pN} / \mathrm{nm})$ & 15.4 & 8.0 & 20.5 & 12.7 & 8.8 & 3.8 \\
\hline$\sigma^{*} A_{\mathrm{p}}(\mathrm{kcal} / \mathrm{mol})$ & 3.9 & 2.8 & 7.0 & 4.1 & 2.4 & 1.1 \\
\hline$\sigma^{*} \beta A_{\mathrm{p}}(\mathrm{kcal} / \mathrm{mol})$ & -8.9 & -2.7 & -5.4 & -1.6 & 0.6 & 0.3 \\
\hline
\end{tabular}

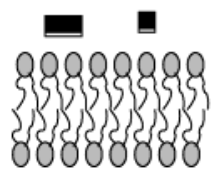

(a)

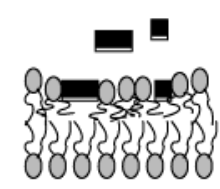

(b)

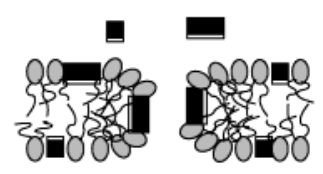

(c)
FIGURE 7: Cartoon of peptide-lipid interactions. The black rectangles represent helical peptides viewed either along the helix or from the side: (a) before binding; (b) binding at $P / L<(P / L)^{*}$; (c) binding at $P / L>(P / L)^{*}$.

peptide. In a bilayer, the chains are the elastic region. A membrane area increase, $\triangle A$, causes a thinning of the chain region, $-\Delta h$, by the relation $-\Delta h / h=\Delta A / A$. Indeed, we have found that in all cases the bilayer thickness decreases linearly with increasing $P / L(34,37,38,42,53)$ until $P / L$ reaches a critical value, $(P / L)^{*}$. Thus the slope of the membrane thickness versus $P / L$ below $(P / L)^{*}$ (Figure 6) gives the value of $A_{\mathrm{P}}$ (Table 1).

It is interesting to note that the measured values for $A_{\mathrm{P}}$ are smaller than the cross sections of the peptides. The lengthwise cross section of the melittin helix has been measured by crystallography (Terwilliger et al. (71), who took into account the solvent content) to be approximately $400 \AA^{2}$, while the monolayer study (72) gave a cross section of $368 \AA^{2}$. The lengthwise cross section for the alamethicin helix is $331 \AA^{2}$ in crystals (73). Corrected for the $30 \%$ solvent content, the alamethicin cross section is $232 \AA^{2}$. The smaller values for $A_{\mathrm{P}}$ as compared with the peptide cross section can be explained if some water molecules are released from the headgroup region when the peptide is embedded. The width of a peptide helix is about $10 \AA$ (35). Thus each alamethicin binding causes release of 8-13 water molecules. In contrast, each melittin binding causes release of 45-70 water molecules. In the following, binding in the headgroup region on the bilayer surface is sometimes referred to as "surface binding" for short, in contrast to binding to the rim of a pore (see cartoons in Figure 7).

2. Threshold (or Critical) Peptide Concentration for Pore Formation, $P / L^{*}$. The concentration dependence of peptide orientation in lipid bilayers was first discovered in the alamethicin/DPhPC system (9). Since then every peptide has shown threshold concentrations $(P / L)^{*}$ where the peptide starts to change orientation in bilayers as $P / L$ further increases, including the $\beta$-sheet peptide protegrin (74). Significantly, neutron diffraction detected transmembrane pores only in the region $P / L>(P / L)^{*}$; no pores were detected for $P / L<(P / L)^{*}(12,13,35,75)$. Thus we have correlated the threshold concentration for peptide orientation change with the threshold concentration for pore formation. Furthermore, we have found that the $(P / L)^{*}$ determined by OCD is coincident with the threshold peptide concentration where the bilayer thickness starts to level off as a function of $P / L$ (34 and Figure 6). The values for $(P / L)^{*}$ in Table 1 were determined from the plot $\phi$ versus $1 /(P / L)$ where the threshold for peptide orientation change is clearly defined (33; see Figure 2$)$. The fact that $(P / L)^{*}$ varies greatly with the lipid composition of the bilayer may be closely related to the varying efficacy of the peptides against different cell types.

The lipids for this study were chosen for having the critical peptide concentration $P / L^{*}$ within the measurable range of OCD and X-ray. The sensitivities of OCD measurement for peptide orientation change and diffraction measurement for membrane thickness change are both limited to $P / L$ larger than $1 / 200$. For example, lipids with unbranched, saturated chains have undetectably low $(P / L)^{*}$. Both alamethicin and melittin are inserted perpendicularly in dimyristoyl phosphatidylcholine (DMPC) bilayers for all peptide concentrations measurable, as long as the bilayers are in the fluid phase; hence DMPC is not included in this study. We were tempted to find a relation between $(P / L)^{*}$ and the headgroup size relative to the lipid cross section area. For example, DPhPC has the largest cross section $A_{\mathrm{L}}$ among the PC lipids, and it also has the highest $(P / L)^{*}$ for both melittin and alamethicin. If we introduce $A_{\mathrm{H}}$ as the cross section of the lipid headgroup, one might qualitatively interpret this result as due to DPhPC having the largest $\left(A_{\mathrm{L}}-A_{\mathrm{H}}\right)$ or the smallest $A_{\mathrm{H}} / A_{\mathrm{L}}$ ratio. In a previous study (38), we have shown that in $\mathrm{DPhPC} / \mathrm{DPhPE}$ mixture bilayers, the $(P / L)^{*}$ increased linearly with the PE content, and we explained the result in terms of the average $A_{\mathrm{H}} / A_{\mathrm{L}}$ ratio. In the present study, ( $P /$ $L)^{*}$ of alamethicin in DOPC is below $1 / 200$. That is why we chose DOPC/DOPE 2:1 mixture to raise the $(P / L)^{*}$ to the measurable range of $1 / 187$.

However, this simplistic reasoning breaks down in the case of melittin/DOPC. The lipid cross section $A_{\mathrm{L}}$ of DOPC is between that of POPC or DiC22:1PC and that of DPhPC. Therefore one would expect a $(P / L)^{*}$ between $1 / 30$ (for $\mathrm{DPhPC}$ ) and 1/60 (estimated for POPC or DiC22:1PC), but instead the $(P / L)^{*}$ for DOPC is $1 / 99$. This tells us that there are other nonobvious factors about lipids that significantly affect the peptide-lipid interactions.

One noticeable difference between alamethicin and most other charged antimicrobial peptides (including melittin) is that the threshold value $(P / L)^{*}$ for alamethicin is rather sensitive to the degree of hydration for lipids. For example, 
in alamethicin/DPhPC, $(P / L)^{*}=1 / 58$ at $98 \% \mathrm{RH}$, whereas $(P / L)^{*}=1 / 118$ at full hydration (33). For highly charged antimicrobial peptides, $(P / L)^{*}$ is not sensitive to the level of hydration.

3. Tension for Pore Formation, $\sigma^{*}$. Membrane area expansion $(\triangle A)$ and membrane tension $(\sigma)$ are related by $\sigma$ $=K_{\mathrm{A}} \Delta A / A$. Thus the area expansion induced by peptide embedment creates a stress equivalent to a membrane tension. We shall call this an internal membrane tension because it does not exert a force at the membrane boundary. However, its value should be similar to the external tension required to stretch the membrane area by the same fraction of $\triangle A / A$. Since pores did not appear until $P / L$ reached $(P / L)^{*}$, we consider the tension at $P / L=(P / L)^{*}$ the tension required for pore formation, $\sigma^{*}=K_{\mathrm{A}}(\Delta A / A)_{\max }=K_{\mathrm{A}}\left(A_{\mathrm{P}} / A_{\mathrm{L}}\right)\left((P / L)^{*}\right)$ (Table 1). These values are to be compared with the rupture tensions for single vesicles. Olbrich et al. (76) used micropipet aspiration to test the mechanical strength of lipid vesicles. For lipids of 18-carbon chains with one to four cisdouble bonds, the tensions for vesicle rupture were measured to be in the range from 3 to $10 \mathrm{pN} / \mathrm{nm}$ (76). It is reasonable to assume that the vesicle rupture started with the formation of a pore. Our values for the tension of pore formation, $\sigma^{*}$, range from 4 to $21 \mathrm{pN} / \mathrm{nm}$ (Table 1). DOPC was measured in both experiments: the rupture tension is $9.9 \pm 2.6 \mathrm{pN} /$ $\mathrm{nm}$ in agreement with our $\sigma^{*}=8.0 \mathrm{pN} / \mathrm{nm}$. $\sigma^{*}$ for other lipids are also in the same range of values as those for the rupture tensions. We take this as a strong indication that peptide-induced pores are caused by an internal membrane tension. In agreement with this interpretation is the observation that the lipid vesicle with bound peptides requires a smaller external tension to reach the point of rupture (65). It is as if the tension for rupture is the same for pure lipid vesicles and for vesicles with bound peptides, except for the latter the tension for rupture is the sum of the peptide-induced internal tension and the external tension.

(4) The Effect of Pores on Membrane Thickness, $\beta$. Consider the free energy change when a peptide binds to a lipid bilayer from solution. Before the bound peptide-to-lipid ratio $P / L$ exceeds the threshold $(P / L)^{*}$, all peptides are bound in the headgroup region on the membrane surface, which has the effect of increasing the membrane area. Therefore the differential free energy change can be written as $\delta F=$ $-\epsilon_{\mathrm{s}} \delta P+\sigma \delta A$, where $\epsilon_{\mathrm{s}}$ is the binding energy. For a finite (rather than infinitesimal) $P / L$, the free energy change normalized to per lipid is

$$
\begin{array}{r}
\Delta F / L=\Delta f=-\epsilon_{\mathrm{s}}(P / L)+{ }^{1} /{ }_{2} K_{\mathrm{A}} A_{\mathrm{L}}(\Delta A / A)^{2}= \\
-\epsilon_{\mathrm{s}}(P / L)+{ }_{1}{ }_{2} K_{\mathrm{A}}\left(A_{\mathrm{P}}{ }^{2} / A_{\mathrm{L}}\right)(P / L)^{2}
\end{array}
$$

For $P / L$ above the threshold, a fraction of the bound peptide molecules, $\phi P \equiv P_{\mathrm{I}}$, participates in pore formation. We have to allow for a possible effect of the pore on the membrane thickness. Thus we modify $\Delta A=A_{\mathrm{P}} P$ for $P / L<$ $(P / L) *$ to

$$
\Delta A=A_{\mathrm{P}}\left(P-P_{\mathrm{I}}\right)+\beta A_{\mathrm{P}} P_{\mathrm{I}}
$$

for $P / L>(P / L)^{*}$. The parameter $\beta$ expresses the effect of a peptide in the pore relative to the effect of a peptide bound on the surface. For example, if $\beta=1$, the pores have the same effect of thinning as if the pore-participating peptides were on the surface, and if $\beta=0$, the pores have no effect on the membrane thickness. Then, for $P / L>(P / L)^{*}$, the free energy change is given by $(33,34)$

$$
\begin{aligned}
\Delta f=-\epsilon_{\mathrm{s}}(1-\phi)(P / L)-\epsilon_{\mathrm{p}} \phi P / L+ \\
{ }_{1}{ }_{2} K_{\mathrm{A}}\left(A_{\mathrm{P}}{ }^{2} / A_{\mathrm{L}}\right)[(1-\phi) P / L+\beta \phi P / L]^{2}
\end{aligned}
$$

$-\epsilon_{\mathrm{p}}$ is the pore energy per peptide. Minimization of $\Delta f$ with respect to $\phi$, that is, $\partial \Delta f / \partial \phi=0$, led to an equation relating the fraction of peptides participating in the pores to $P / L(33)$ :

$$
\phi=\frac{1}{1-\beta}\left(1-\frac{(P / L)^{*}}{P / L}\right)
$$

where the threshold concentration is given by

$$
P / L^{*}=\frac{\epsilon_{\mathrm{s}}-\epsilon_{\mathrm{p}}}{K_{\mathrm{A}}\left(A_{\mathrm{P}}^{2} / A_{\mathrm{L}}\right)(1-\beta)}
$$

The parameter $\beta$ is obtained from the slope of $\phi$ versus $1 /(P / L)$ (Figure 2). Since the binding energy $-\epsilon_{\mathrm{s}}$ is necessarily lower than the pore energy $-\epsilon_{\mathrm{p}}$ (which is why there are no pores at low $P(L)$ or $\epsilon_{\mathrm{s}}>\epsilon_{\mathrm{p}}$, eq 6 implies that $\beta<1$. Interestingly, $\beta$ can be either positive or negative. When $\beta$ is positive, as in the case of alamethicin, the pore formation tends to thin the membrane, although its effect is not as strong as when the peptide is bound on the surface. Since alamethicin forms barrel-stave pores (35), the thinning effect could be due to a hydrophobic mismatch between the hydrophobic region of the alamethicin helix and the hydrocarbon region of the lipid bilayer $(49,50,77,78)$. Presumably the hydrophobic length of the alamethicin helix is shorter than the hydrocarbon thickness of the lipid bilayers (the $h$ value in Table 1). Unfortunately it has not been possible to define the hydrophobic length from examining the amino acid composition of alamethicin helix. This difficulty is illustrated by the example of gramicidin. It is well-known that the gramicidin channel is made of hydrophobic amino acids and has a molecular length of $26 \AA$ (39). But the experiments of membrane thickness effects indicated that its hydrophobic length is only $22 \AA(49,50,77,79)$.

In the case of melittin, $\beta$ is negative. This implies that the pore formation has an effect of thickening, rather than thinning, the bilayer. This might be caused by the bending energy stored in a toroidal pore. The spontaneous curvature of a lipid monolayer consisting of bulky chains is negative, as is the case for all the lipids used in this study. At the rim of a toroidal pore, the principal curvature along the direction normal to the plane of the bilayer is positive. Thus the natural tendency of the negative spontaneous curvature might tend to thicken the bilayer at the edge of the pore. Indeed the lipid with the bulkiest chains, DPhPC, appears to have the most negative $\beta$.

A negative $\beta$ has the consequence of preventing the peptide molecules from all participating in pore formation, or $\phi \rightarrow$ 1 , as can be seen mathematically from eq 5 . This is contrary to the case of positive $\beta$ (alamethicin), where all the peptides form pores at very high concentrations (Figure 2). We can understand this disparity by examining how the peptides bind to the bilayer at $P / L>(P / L)^{*}$. We have shown that at $P / L$ $>(P / L)^{*}$ the binding process takes place at constant $\Delta A$ (eq 3 ), as demanded by minimization of free energy. Take the 
example of melittin binding to DOPC bilayer for which $\beta$ $\approx-1$. For every peptide molecule binding to a pore, another peptide molecule must bind to the membrane surface to keep $\Delta A=A_{\mathrm{P}}\left(P-P_{\mathrm{I}}\right)+\beta A_{\mathrm{P}} P_{\mathrm{I}}$ constant. Consequently there will always be peptides bound to the membrane surface at any $P / L$. In contrast, take the example of alamethicin binding to $\mathrm{DPhPC}$ for which we have $\beta \approx 1 / 4$. For every three peptide molecules binding to pores, one peptide molecule must transfer from the membrane surface to pores to keep $\Delta A=$ $A_{\mathrm{P}}\left(P-P_{\mathrm{I}}\right)+\beta A_{\mathrm{P}} P_{\mathrm{I}}$ constant. Thus eventually all alamethicin molecules will participate in pore formation at a high enough peptide concentration.

5. Binding Energy $\epsilon_{s}$ and Pore Energy $\epsilon_{p}$. The free energy change when a peptide molecule binds to the surface from solution is $-\epsilon_{\mathrm{s}}+\sigma A_{\mathrm{P}}$, whereas the free energy change when a peptide molecule binds to form a pore (at $P / L>(P / L)^{*}$ ) is $-\epsilon_{\mathrm{p}}+\sigma^{*} \beta A_{\mathrm{P}}$. These two free energy changes are equal at $P / L=(P / L)^{*}$ :

$$
-\epsilon_{\mathrm{s}}+\sigma^{*} A_{\mathrm{P}}=-\epsilon_{\mathrm{p}}+\sigma^{*} \beta A_{\mathrm{P}}
$$

In fact eq 7 is another way of deriving eq 6 . Both sides of eq 7 have to be negative, otherwise the peptides will not bind to lipid bilayers from solution. The magnitudes of $\sigma^{*} A_{\mathrm{P}}$ and $\beta \sigma^{*} A_{\mathrm{P}}$ are in the range from -9 to $7 \mathrm{kcal} / \mathrm{mol}$. The negative values of $\beta \sigma^{*} A_{\mathrm{P}}$ represent the tension energies reduced by pore formation (normalized to per peptide).

The binding energy $-\epsilon_{\mathrm{s}}$ is directly measurable, particularly by titration calorimetry $(63,64)$. Its apparent value depends on the charge of the lipid, $\mathrm{pH}$ of the solution, and other factors. Careful analyses can differentiate these factors from the intrinsic partition coefficient of hydrophobic interaction, which is independent of electrostatic effects $(63,64,80,81)$. For example, the binding energies corresponding to the intrinsic (hydrophobic) partition of both melittin and magainin to the neutral lipid POPC are about $-7.0 \mathrm{kcal} / \mathrm{mol}(63,64$, $80,81)$. If the typical binding energy for melittin to neutral lipids were $-7.0 \mathrm{kcal} / \mathrm{mol}$, the left-hand side of eq $7,-\epsilon_{\mathrm{s}}+$ $\sigma^{*} A_{\mathrm{P}}$, would indeed satisfy the condition of being zero or negative for all the lipids that we have studied.

The pore energy $-\epsilon_{\mathrm{p}}$ has not been directly measured so far. However its magnitude relative to the binding energy $\delta \epsilon=\epsilon_{\mathrm{s}}-\epsilon_{\mathrm{p}}$ can be deduced from our measurement according to eq 6 (or equivalently eq 7 ). The results for six peptide/lipid combinations are shown in Table 1. From $-\epsilon_{\mathrm{s}}$ and $\delta \epsilon$, the value of the pore energy $-\epsilon_{\mathrm{p}}$ can be determined. It is noteworthy to point out that $\delta \epsilon$ for alamethicin or barrelstave pores is much smaller than that for melittin or toroidal pores. Again if $-\epsilon_{\mathrm{s}}$ were constant for melittin to neural lipids, the values of $\delta \epsilon$ then reflect how the pore energy depends on the characters of lipids. DOPC and POPC have similar $h$ and $A_{\mathrm{L}}$, they also have similar $\delta \epsilon$. Compared with DOPC and POPC, DPhPC has a similar $h$ but a large $A_{\mathrm{L}}$, whereas DiC22:1PC has a large $h$ but a similar $A_{\mathrm{L}}$. The latter two lipids end up having a similarly larger $\delta \epsilon$. These numbers should be useful for further analyses by molecular models.

The detailed analysis for the pore energy is very complex $(30,31)$. Nevertheless we can qualitatively understand why pores induced by peptides are stable. Note that in the pore energy $E_{\mathrm{R}}=\gamma 2 \pi R-\sigma \pi R^{2}$ the tension $\sigma$ is proportional to $\Delta A=A_{\mathrm{P}}\left[P-(1-\beta) P_{\mathrm{I}}\right]($ eq 3$)$ where $(1-\beta)>0$. Thus transferring a peptide from surface binding to pore binding

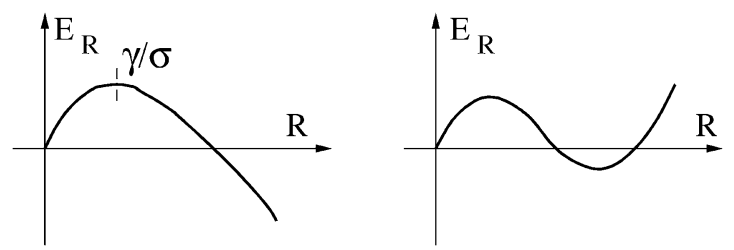

FIGURE 8: A schematic curve for (left) $E_{\mathrm{R}}=\gamma 2 \pi R-\sigma \pi R^{2}$, where no pore is stable, and (right) $E_{\mathrm{R}}=c_{1} R-c_{2} R^{2}+c_{3} R^{3}$ (all coefficients $c_{i}>0$ ), where a stable pore is possible.

reduces the tension. Assuming that the line density of peptide on the rim of pore is constant (this is true for detergents at high detergent concentrations; see refs 30 and 31 ), then the number $P_{\mathrm{I}}$ is proportional to $R$. Therefore the tension has the following $R$-dependence $\sigma=\sigma_{\mathrm{o}}-\alpha R$ (with a positive constant $\alpha$ ). We see that membrane tension $\sigma$ tends to enlarge $R$ (lowering the energy $E_{\mathrm{R}}$ ), but an enlarged $R$ will reduce $\sigma$. This feedback mechanism is what makes the pore radius stable. The complete $R$-dependence of the pore energy has the form $E_{\mathrm{R}}=c_{1} R-c_{2} R^{2}+c_{3} R^{3}$ (all coefficients $c_{i}>0$ ), which makes a stable pore possible (Figure 8). A more realistic analysis of pore energy is beyond the scope of this paper. It will be discussed elsewhere.

\section{CONCLUDING REMARK: CELL TYPE SELECTIVITIES OF ANTIMICROBIAL PEPTIDES}

This paper presents our attempt to describe in terms of quantitative parameters the peptide-lipid interactions related to the mechanism of pore formation. Experiments have shown that the threshold peptide concentration for pore formation varies with peptide and also with the lipid composition. These variations with peptides and with lipids could be a reason for the different minimum inhibitory concentrations (MIC) exhibited by different antimicrobial peptides in the same bactericidal assays, and for the different MIC exhibited by the same peptide against different pathogens (21). By the quantitative parameters, we hope to take the pore forming activities of the peptides one step closer to a molecular description of the process. These parameters should be useful for further molecular analyses and for molecular dynamic simulation studies.

One important problem for antimicrobial peptides is to understand the basis for the cell type selectivities of the peptides. A key distinction between bacterial membranes and the membranes of eukaryotic cells is the presence of negatively charged lipid headgroups in the outermost leaflet of the former but not in the latter. Most antimicrobial peptides are highly cationic. The electrostatic attraction of the peptides to the bacterial surfaces makes the density of bound peptides $(P / L)$ in bacterial membranes much higher than that in animal cell membranes (63), if both membranes are presented to the same solution of peptides. Since the pore formation takes place only if $P / L$ exceeds a threshold value $(P / L)^{*}$, the effect of the charge alone seems to explain why these peptides are effective antibiotics and at the same time noncytolytic to the host cells

However, detailed analyses found that different peptides preferentially killed different pathogens and exhibited varying degrees of hemolytic activities $(82,83)$. This cannot be explained by the effect of electrostatic attraction. We should also keep in mind a caveat when we compare the results between experiments with model membranes on one hand 
and activity assays on cells on the other. In experiments with model membranes, the peptide concentration $P / L$ tends to be uniform over the entire membrane, but in activity assays on cells, a high local peptide concentration on the cell membrane can determine the fate of the cell. The recent discovery of high-affinity binding of $\theta$-defensins to glycoproteins and glycolipids (57) points to a possibility of local accumulation of peptides on the membrane surface. Antimicrobial peptides clearly work by high concentration. Thus they target those membranes that accumulate them, either by charge or by docking sites. However the details must also depend on the lipid dependence suggested by the examples observed here. A systematic analysis of peptides and lipids and elucidation of the molecular compositions of cell membranes are required to solve the riddle of cell type selectivity.

\section{ACKNOWLEDGMENT}

H.W.H. thanks Robert I. Lehrer for discussions.

\section{REFERENCES}

1. Meyer, P., and Reusser, F. (1967) A polypeptide antibacterial agent isolated from Trichoderma viride, Experientia 23, 85-86.

2. Mueller, P., and Rudin, D. O. (1968) Action potential induced in biomolecular lipid membranes, Nature 217, 713-719.

3. Latoree, R., and Alvarez, O. (1981) Voltage-dependent channels in planar lipid bilayer membranes, Physiol. Rev. 61, 77-150.

4. Fringeli, U. P., and Fringeli, M. (1979) Pore formation in lipid membranes by alamethicin, Proc. Natl. Acad. Sci. U.S.A. 76, 3852-3856.

5. LaTorre, R., Miller, C. G., and Quay, S. (1981) Voltage-dependent conductance induced by alamethicin-phospholipid conjugates in lipid bilayers, Biophys. J. 36, 803-809.

6. Knoll, W. (1986) Raman spectroscopic evidence for the incorporation of alamethicin into dimyristoylphosphatidylcholine bilayers, Biochim. Biophys. Acta 863, 329-331.

7. Schwarz, G., Stankowski, S., and Rizzo, V. (1986) Thermodynamic analysis of incorporation and aggregation in a membrane: application to the pore-forming peptide alamethicin, Biochim. Biophys. Acta 861, 141-151.

8. Rizzo, V., Stankowski, S., and Schwarz, G. (1987) Alamethicin incorporation in lipid bilayers: a thermodynamics study, Biochemistry 26, 2751-2759.

9. Huang, H. W., and Wu, Y. (1991) Lipid-alamethicin interactions influence alamethicin orientation, Biophys. J. 60, 1079-1087.

10. Olah, G. A., and Huang, H. W. (1988) Circular dichroism of oriented $\alpha$-helices. I. proof of the exciton theory, J. Chem. Phys. $89,2531-2538$.

11. Wu, Y., Huang, H. W., and Olah, G. A. (1990) Method of oriented circular dichroism, Biophys. J. 57, 797-806.

12. He, K., Ludtke, S. J., Worcester, D. L., and Huang, H. W. (1995) Antimicrobial peptide pores in membranes detected by neutron in-plane scattering, Biochemistry 34, 16764-16769.

13. He, K., Ludtke, S. J., Worcester, D. L., and Huang, H. W. (1996) Neutron scattering in the plane of membranes: structure of alamethicin pores, Biophys. J. 70, 2659-2666.

14. Habermann, E. (1972) Bee and wasp venom: the biochemistry and pharmacology of their peptides and enzymes are reviewed, Science 177, 314-322.

15. Zasloff, M. (1987) Magainins, a class of antimicrobial peptides from Xenopus skin: isolation, characterization of two active forms, and partial cDNA sequence of a precursor, Proc. Natl. Acad. Sci. U.S.A. 84, 5449-5453.

16. Katsu, T., Ninomiya, C., Kuroko, M., Kobayashi, H., Hirota, T., and Fujita, Y. (1988) Action mechanism of amphipathic peptides gramicidin $\mathrm{S}$ and melittin on erythrocyte membrane, Biochim. Biophys. Acta 939, 57-63.

17. Ladokhin, A. S., Selsted, M. E., and White, S. H. (1997) Sizing membrane pores in lipid vesicles by leakage of co-encapsulated markers: pore formation by melittin, Biophys. J. 72, 1762-1766.

18. Matsuzaki, K., Yoneyama, S., and Miyajima, K. (1997) Pore formation and translocation of melittin, Biophys. J. 73, 831-838.
19. Matsuzaki, K., Harada, M., Handa, T., Funakoshi, S., Fujii, N., Yajima, H., and Miyajima, K. (1989) Magainin 1-induced leakage of entrapped calcein out of negatively-charged lipid vesicles, Biochim. Biophys. Acta 981, 130-134.

20. Matsuzaki, K., Murase, O., and Miyajima, K. (1995) Kinetics of pore formation by an antimicrobial peptide, magainin 2 , in phospholipid bilayers, Biochemistry 34, 12553-12559.

21. Boman, H. G., Marsh, J., and Goode, J. A., Eds. (1994) Antimicrobial Peptides, Antimicrobial Peptides, Ciba Foundation Symposium 186, pp 1-272, John Wiley \& Sons, Chichester, U.K.

22. Matsuzaki, K. (1998) Magainins as paradigm for the mode of action of pore forming polypeptides, Biochim. Biophys. Acta 1376, 391-400.

23. Huang, H. W. (2000) Action of antimicrobial peptides: Two-state model, Biochemistry 39, 8347-8352.

24. Zasloff, M. (2002) Antimicrobial peptides of multicellular organisms, Nature 415, 389-395.

25. Litster, J. D. (1975) Stability of lipid bilayers and red blood cell membranes, Phys. Lett. 53A, 193-194.

26. Taupin, C., Dvolaitzky, M., and Sauterey, C. (1975) Osmotic pressure induced pores in phospholipid vesicles, Biochemistry 14 , $4771-4775$.

27. Kabalnov, A., and Wennerstrom, H. (1996) Macroemulsion stability: the oriented wedge theory revisited, Langmuir 12, 276292.

28. Weaver, J. C., and Chizmadzhev, Y. A. (1996) Theory of electroporation: a review, Bioelectrochem. Bioenerg. 41, 135160.

29. Brochard-Wyart, F., de Gennes, P. G., and Sandre, O. (2000) Transient pores in stretched vesicles: role of leak out, Physica A 278, 32-51.

30. Karatekin, E., Sandre, O., Guitouni, H., Borghi, N., Puech, P.-H., and Brochard-Wyart, F. (2003) Cascades of transient pores in giant vesicles: line tension and transport, Biophys. J. 84, 1734-1749.

31. Puech, P.-H., Borghi, N., Karatekin, E., and Brochard-Wyart, F. (2003) Line thermodynamics: adsorption at a membrane edge, Phys. Rev. Lett. 12, 128304-1-128304-4.

32. Melikov, K. Ch., Frolov, V. A., Shcherbakov, A., Samsonov, A. V., Chizmadzhev, Y. A., and Chernomordik, L. V. (2001) Voltageinduced nonconductive pre-pores and metastable single pores in unmodified planar lipid bilayer, Biophys. J. 80, 1829-1836.

33. Chen, F. Y., Lee, M. T., and Huang, H. W. (2002) Sigmoidal concentration dependence of antimicrobial peptide activities: a case study on alamethicin, Biophys. J. 82, 908-914.

34. Chen, F. Y., Lee, M. T., and Huang, H. W. (2003) Evidence for membrane thinning effect as the mechanism for peptide-induced pore formation, Biophys. J. 84, 3751-3758.

35. Yang, L., Harroun, T. A., Weiss, T. M., Ding, L., and Huang, H. W. (2001) Barrel-stave model or toroidal model? A case study on melittin pores, Biophys. J. 81, 1475-1485.

36. Pandey, R. C., Cook, J. C., and Rinehart, K. L. (1977) High resolution and field desorption mass spectrometry studies and revised structure of alamethicin I and II, J. Am. Chem. Soc. 99, 8469-8483.

37. Wu, Y, He, K., Ludtke, S. J., and Huang, H. W. (1995) X-ray diffraction study of lipid bilayer membrane interacting with amphiphilic helical peptides: diphytanoyl phosphatidylcholine with alamethicin at low concentrations, Biophys. J. 68, 23612369.

38. Heller, W. T., He, K., Ludtke, S. J., Harroun, T. A., and Huang, H. W. (1997) Effect of changing the size of lipid headgroup on peptide insertion into membranes, Biophys. J. 73, 239-244.

39. Olah, G. A., Huang, H. W., Liu, W., and Wu, Y. (1991) Location of ion binding sites in the gramicidin channel by X-ray diffraction, J. Mol. Biol. 218, 847-858.

40. Chen, F. Y., Hung, W. C., and Huang, H. W. (1997) Critical swelling of phospholipid bilayers, Phys. Rev. Lett. 79, 40264029.

41. Hung, W. C., Chen, F. Y., and Huang, H. W. (2000) Orderdisorder transition in bilayers of diphytanoyl phosphatidylcholine, Biochim. Biophys. Acta 1467, 198-206.

42. Ludtke, S., He, K., and Huang, H. W. (1995) Membrane thinning caused by magainin 2, Biochemistry 34, 16764-16769.

43. Blaurock, A. E. (1971) Structure of the nerve myelin membrane: proof of the low resolution profile, J. Mol. Biol. 56, 35-52.

44. Guinier, A. (1994) X-ray diffraction in crystals, imperfect crystals, and amorphous bodies, pp 151-251, Dover Publications, New York. 
45. Perutz, M. F. (1954) The structure of haemoglobin. III. Direct determination of the molecular transform, Proc. R. Soc. London, Ser. A 225, 264-286.

46. Torbet, J., and Wilkins, M. H. F. (1976) X-ray diffraction studies of lecithin bilayers, J. Theor. Biol. 62, 447-458.

47. McIntosh, T. J., and Simon, S. A. (1986) Area per molecule and distribution of water in fully hydrated dilauroylphosphatidylethanolamine bilayers, Biochemistry 25, 4948-4952.

48. Nagle, J. F., and Tristram-Nagle, S. (2000) Structure of lipid bilayers, Biochim. Biophys. Acta 1469, 159-195.

49. Harroun, T. A., Heller, W. T., Weiss, T. M., Yang, L., and Huang, H. W. (1999) Experimental evidence for hydrophobic matching and membrane-mediated interactions in lipid bilayers containing gramicidin, Biophys. J. 76, 937-945.

50. Harroun, T. A., Heller, W. T., Weiss, T. M., Yang, L., and Huang, H. W. (1999) Theoretical analysis of hydrophobic matching and membrane-mediated interactions in lipid bilayers containing gramicidin, Biophys. J. 76, 3176-3185.

51. Hung, W. C., and Chen, F. Y. (2003) The hydrophobichydrophilic interface of phospholipid membranes studied by lamellar X-ray diffraction, Chin. J. Phys. 41, 85-91.

52. Rawicz, W., Olbrich, K. C., McIntosh, T., Needham, D., and Evans, E. (2000) Effect of chain length and unsaturation on elasticity of lipid bilayers, Biophys. J. 79, 328-339.

53. Heller W. T., Waring, A. J., Lehrer, R. I., Harroun, T. A., Weiss, T. M., Yang, L., and Huang, H. W. (2000) Membrane thinning effect of the $\beta$-sheet antimicrobial protegrin, Biochemistry 39, 139-145.

54. Weiss, T. M., Yang, L., Ding, L., Waring, A. J., Lehrer, R. I., and Huang, H. W. (2002) Two states of cyclic antimicrobial peptide RTD-1 in lipid bilayers, Biochemistry 41, 10070-10076.

55. Tang, Y. Q., Yuan, J., Osapay, G., Osapay, K., Tran, D., Miller, C. J., Ouellette, A. J., and Selsted, M. E. (1999) A cyclic antimicrobial peptide produced in primate leukocytes by the ligation of two truncated alpha-defensins, Science 286, 498-502.

56. Tran, D., Tran, P. A., Tang, Y.-Q., Yuan, J., Cole, T., and Selsted M. E. (2002) Homodimeeric $\theta$-defensins from Rhesus macaque leukocytes, J. Biol. Chem. 277, 3079-3084.

57. Wang, W., Cole, A. M., Hong, T., Waring, A. J., and Lehrer, R. I. (2003) Retrocyclin, an antiretroviral $\theta$-defensin, is a lectin, $J$. Immunology 170, 4708-4716.

58. Hirsh, D. J., Hammer, J., Maloy, W. L., Blazyk, J., and Schaefer, J. (1996) Secondary structure and location of a magainin analogue in synthetic phospholipid bilayers, Biochemistry 35, 12733-12741.

59. Bechinger, B., Kim, Y., Chirlian, L. E., Gesell, J., Neumann, J.M., Motal, M. Tomich, J., Zasloff, M., and Opella, S. J. (1991) Orientations of amphipathic helical peptides in membrane bilayers determined by solid-state NMR spectroscopy, J. Biomol. NMR 1 , $167-173$

60. Williams, R. W., Starman, R., Taylor, K. M. P., Cable, K., Beeler, T., Zasloff, M., and Covell, D. (1990) Raman spectroscopy of synthetic antimicrobial frog peptides magainin 2a and PGLa, Biochemistry 29, 4490-4496.

61. Matsuzaki, K., Murase, O., Tokuda, H., Fumakoshi, S., Fujii, N., and Miyajima, K. (1994) Orientational and aggregational states of magainin 2 in phospholipid bilayers, Biochemistry 33, 33423349.

62. Matzuzaki, K., Harada, M., Fumakoshi, S., Fujii, N., and Miyajima, K. (1991) Physicochemical determinants for the interactions of magainins 1 and 2 with acidic lipid blayers, Biochim. Biophys. Acta 1063, 162-170.

63. Wenk, M. R., and Seelig, J. (1998) Magainin 2 amide interaction with lipid membranes: calorimetric detection of peptide binding and pore formation, Biochemistry 37, 3909-3916.
64. Wieprecht, T., Beyermann, M., and Seelig, J. (1999) Binding of antimicrobial magainin peptides to electrically neutral membranes: thermodynamics and structure, Biochemistry 38, 1037710387.

65. Longo, M. L., Waring, A. J., Gordon, L. M., and Hammer, D. A. (1998) Area expansion and permeation of phospholipid membrane bilayers by influenza fusion peptides and melittin, Langmuir 14, $2385-2395$.

66. Huang, H. W. (1995) Elasticity of lipid bilayer interaction with amphiphilic helical peptides, J. Phys. II Fr. 5, 1427-1431.

67. Gazit, E., Lee, W. J., Brey, P. T., and Shai, Y. (1994) Mode of action of the antibacterial Cecropin B2: a spectrofluometric study, Biochemistry 33, 10681-10692.

68. Gazit, E., Boman, A., Boman, H., and Shai, Y. (1995) Interaction of the mammalian antibacterial peptide Cecropin P1 with phospholipid vesicles, Biochemistry 34, 11479-11488.

69. Schümann, M., Margitta, D., Wieprecht, T., Beyermann, M., and Bienert, M. (1997) The tendency of magainin to associate upon binding to phospholipid bilayers, Biochemistry 36, 4345-4351.

70. Barranger-Mathys, M., and Cafiso, D. S. (1993) Collisions between helical peptides in membranes monitored using electron paramagnetic resonance: evidence that alamethicin is monomeric in the absence of a membrane potential, Biophys. J. 67, 172-176.

71. Terwilliger, T. C., Weissman, L., and Eisenberg, D. (1982) The structure of melittin in the form I crystals and its implication for melittin's lytic and surface activities, Biophys. J. 37, 353-361.

72. DeGrado, W. F., Kezdy, F. J., and Kaiser, E. T. (1981) Design, synthesis and characterization of a cytotoxic peptide with melittinlike activity, J. Am. Chem. Soc. 103, 679-681.

73. Fox, R. O., and Richards, F. M. (1982) A voltage-gated ion channel model inferred from the crystal structure of alamethicin at $1.5-\AA$ resolution, Nature 300, 325-330.

74. Heller, W. T., Waring, A. J., Lehrer, R. I., and Huang, H. W. (1998) Multiple states of $\beta$-sheet peptide protegrin in lipid bilayers, Biochemistry 37, 17331-17338.

75. Ludtke, S. J., He, K., Heller, W. T., Harroun, T. A., Yang, L., and Huang, H. W. (1996) Membrane pores induced by magainin, Biochemistry 35, 13723-13728.

76. Olbrich, K., Rawicz, W., Needham, D., and Evans, E. (2000) Water permeability and mechanical strength of polyunsaturated lipid bilayers, Biophys. J. 79, 321-327.

77. Huang, H. W. (1986) Deformation free energy of bilayer membrane and its effect on gramicidin channel lifetime, Biophys. J. 50, 1061-1070.

78. Weiss, T. M., van der Wel, P. C. A., Killian, J. A., Koeppe, R. E., II, and Huang, H. W. (2003) Hydrophobic mismatch between helices and lipid bilayers, Biophys. J. 84, 379-385.

79. Elliott, J. R., Needham, D., Dilger, J. P., and Hayden, D. A. (1983) The effects of bilayer thickness and tension on gramicidin singlechannel lifetime, Biochim. Biophys. Acta 735, 95-103.

80. Kuchinka, E., and Seelig, J. (1989) Interaction of melittin with phosphatidylcholine membranes. Binding isotherm and lipid headgroup conformation, Biochemistry 28, 4216-4221.

81. Beschiaschvili, G., and Seelig, J. (1990) Melittin binding to mixed phosphatidylglycerol/phosphatidylcholine membranes, Biochemistry 29, 52-58.

82. Steiner, H., Andreu, D., and Merrifield, R. B. (1988) Binding and action of cecropin and cecropin analogues: antibacterial peptides from insects, Biochim. Biophys. Acta 939, 260-266.

83. Hoffmann, J. A., Kafatos, F. C., Janeway, C. A., Jr., and Ezekowitz, R. A. B. (1999) Phylogenetic perspectives in innate immunity, Science 284, 1313-1318.

BI036153R 